\title{
From Iterative Balance Models to Directly Calculating Explicit Models for Real-time Process Optimization and Scheduling
}

\author{
Tomas Björkqvist $^{1} \quad$ Olli Suominen $^{1} \quad$ Matti Vilkko $^{1} \quad$ Mikko Korpi $^{2}$ \\ ${ }^{1}$ Department of Automation Science and Engineering, Tampere University of Technology, Finland, \\ \{tomas.bjorkqvist, olli.suominen, matti.vilkko\} @tut.fi \\ ${ }^{2}$ Research Center Pori, Outotec Oy, Finland, mikko.korpi@outotec.com
}

\begin{abstract}
Optimal utilization of complex processes involves realtime operational optimization and scheduling, especially in cases where the production line consists of both continuous and batch operated unit processes. This kind of real-time optimization requires process models which can be computed significantly faster than realtime. Iterative balance calculation is typically far too slow for these cases. This paper presents a method for converting an iterative balance model to a directly calculating model suitable for on-line process optimization. The approach is demonstrated with the first unit process in the copper smelting line, the flash smelting furnace (FSF). The method consisted of formulating an equation group based on the constrained FSF HSC-Sim model and solving the unknown parameters and static states with use of a symbolic calculation software. The solution was implemented as a function whose calculation time fulfilled the requirements for scheduling use.
\end{abstract}

Keywords: $\quad$ real-time model, static, mass balance, equation group, symbolic computation, metallurgy, copper smelting, scheduling

\section{Introduction}

The general digitalization of society has brought on a pronounced digitalization wave in process industry. The benefits of digitalization are not fully utilized in some conventional industrial processes and there new advantages are available which can improve their efficiency and ability to stay competitive in increasing global competition. Often the design of these industrial processes is based on long term empirical and theoretical knowledge which has been incorporated into thoroughly built mathematical models. These models often include iterative balance calculations to fulfill empirical and physical process constrains. These models are well suited for steady state process design and often used when offering, planning and constructing new process lines.

Optimal utilization of processes typically involves real-time operational optimization and scheduling, especially in cases where the production line consists of both continuous and batch operated unit processes. This kind of real-time optimization requires process models which can be computed significantly faster than realtime. Iterative balance calculation is often far too slow for these cases. The high demand on execution time can often be compensated by lowering demands on model precision for the real-time operation optimization. Examples of demanding real-time optimization utilized in process design can be found in (Harjunkoski et al., 2016; Touretzky et al., 2016; Pelusi 2012a; Pelusi 2012b).

Good examples of thoroughly built steady state models can be found in metallurgy. Most metallurgical processes are old and have large societal impact which has allowed extensive development work to model process behavior over many decades. These processes comprise complex physical and chemical reactions and modelling has been both theoretical and empirical. To fulfill the basic requirement of mass and energy conservation and empirical observations iterative calculation is often employed.

The incentive for this study is the need for operational optimization of a copper smelting line. Optimal operation of a copper smelting line is challenging for the operators as the operation is divided into many complex individual sub processes. Plant wide operation is required to maximize production and resource efficiency. Additionally, more challenging ores have to be used to retain economic competitiveness worldwide which increases the need for process optimization. Improved operation of copper smelting can provide improved utilization of different input materials and recyclants. Copper smelters present a challenging optimization problem where the harsh environment can prevent obtaining mineral and operational information, data is highly uncertain or measurements may be severely delayed. A full scale optimization of the complete process line will include a considerable amount of variables and require the consideration of large time horizons. Further, many of the underlying models are nonlinear. Thus, sub processes and the related models should be relatively lightweight in terms of their computational requirements. In principle, the development of optimization for a copper smelting line 
operation consists of modelling of unit processes and designing of optimization / scheduling for the combined unit process models.

Static input output process models can be derived with use of mass and energy balances supplemented with sometimes uncertain process reaction knowledge completed with empirical knowledge. In principle this empirical knowledge can be written as constraints in equation form. These equations can be completed with mass and energy balances to form a complete equation group determining process reactions. By solving the equation group, the unknown parameters and thereby the static process state can be solved under the given constraints. In practice this approach is challenging as the equations are often complex and manual solutions may be error prone and exceptionally time consuming. Development of aids for this challenge started in the beginning of the 1970s under the scientific area of symbolic computation. Software programs for manual computation are called computer algebra systems (CAS) and are at present highly developed and even implemented in hand held calculators. These systems include Mathematica (Wolfram) and Maple (Maplesoft), the latter has been implemented in Matlab (Mathworks) as the Symbolic Math Toolbox. In later Matlab versions, the toolbox is based on the MuPAD symbolic engine originally developed at the University of Paderborn. Matlab offers a convenient way of shifting from symbolic calculus to numeric powerful computation.

Utilization of symbolic computation for solving unknown variables of restricted mass balance equations seems to be a rare approach or rarely reported. A similar method was used in (Korpela et al., 2014) in the same research group but the authors have not found similar work by others. Symbolic computation is, however, commonly utilized when forming first principle models (Belkhir et al., 2015; Lin et al., 2009; Yakhno et al., 2016). Its use is especially convenient for model design with e.g. Lagrangian mechanics (Moosavian et al., 2004).

For optimization of the operation of the copper smelting line computationally lightweight models of all unit processes are required. This paper presents a method for converting an iterative balance model to a directly calculated model suitable for process operation optimization. The method is demonstrated with the first unit process in the copper smelting line, the flash smelting furnace (FSF).

\section{Copper Production Line}

Copper smelting begins from the mixing of a suitable concentrate mix with a copper content of $20-30 \%$ which, after drying, is fed to the FSF. The mix reacts with the oxygen-enriched air feed and separates to matte $(\sim 62-70 \% \mathrm{Cu})$ and slag. These are removed intermittently from the FSF, matte is moved to the converters, and slag is processed further in the slag treatment plant. After treatment, both FSF and converter slag can be recycled back to the FSF. The matte copper content can be viewed as one of the main decision variables in smelting as the higher copper content in matte is, the higher the copper content in the slag. Additionally, it is often used as a variable in separation of other valuable metals to both matte and slag. Silica flux is added to the FSF feed and to converters during operation to achieve suitable conditions for separation of matte and slag. One of the main bottlenecks for operation is the capacity of the gas treatment plant which produces sulphuric acid from the off gasses of both the FSF and converters.

Pierce-Smith converters use a submerged feed of oxygen enriched air. Converters are operated in batches where first, in multiple slag-making stages, FSF matte is added between air blows. Here, most of the iron compounds will react and move to slag. Second, in one longer copper-making stage the remaining sulphur is removed from copper compounds. Temperature is controlled with the addition of recycled material, e.g. scrap metal. Finally, the ensuing blister copper ( 99\% $\mathrm{Cu})$ is moved to anode furnaces where oxygen is removed from the matte and copper is cast to anodes for transportation to electrolysis. Figure 1 shows a full copper production line including both smelting and refining. A detailed description of the smelting process can be found for example in (Schlesinger et al., 2011).

\section{Model Conversion}

The method for converting an iterative balance model to a directly calculating model is here demonstrated with a model of the flash smelting furnace, modelled in HSCSim (Outotec). HSC-Sim is a calculation module of HSC Chemistry software developed by Outotec. The name refers to the automatically utilized thermochemical database which contains enthalpy $(\mathrm{H})$, entropy (S) and heat capacity $(\mathrm{Cp})$ data for an extensive amount of chemical compounds. The HSC-Sim module enables application of HSC Chemistry to a whole process made up of process units and streams. The HSCSim module consists of a graphical flowsheet and spreadsheet type process unit models. The custom-made variable list enables creation of different types of process models in chemistry, metallurgy, mineralogy, economics, etc. Each process unit is actually one Excel file. In the Distribution units the compounds are divided into elements and calculation is done with element distribution coefficients. Based on process knowledge some coefficients are defined as fixed. Coefficients for assisting elements in compound formation are calculated based on molar need and supply and called float. Surplus elements are divided with coefficients called rest. Units can be used together or separately and the calculations can be Excel- or DLL-based. 


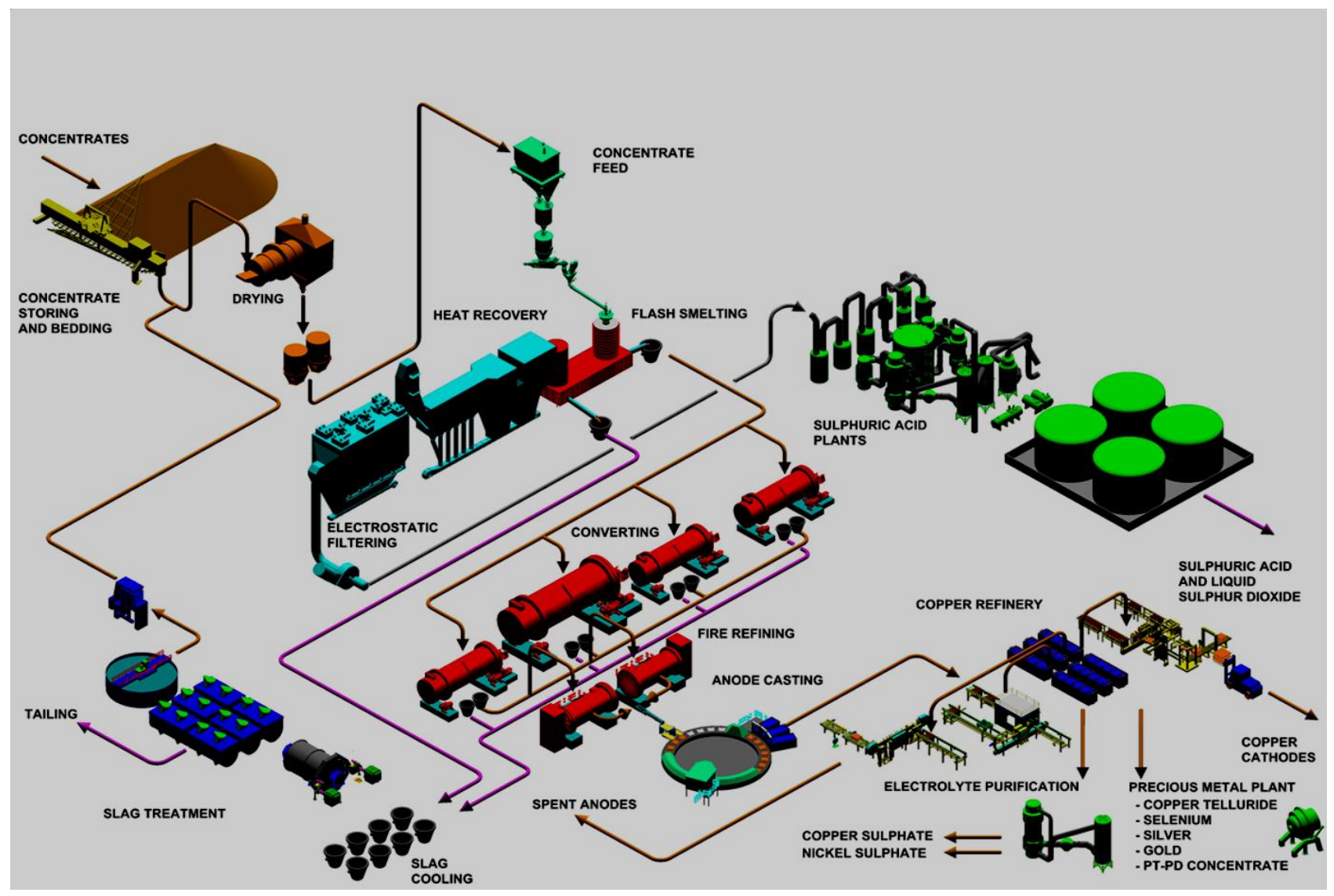

Figure 1. Flow sheet of copper process at Boliden Harjavalta (Boliden).

HSC Sim pyro models are mathematical process models based on mass- and energy balances and empirical knowledge controlling the equilibrium state. These models are successfully used in strategic planning of metal processing. The drawback of these models is the iterative calculation needed for reaching the equilibrium state. This iterative calculation is too slow for use in on-line process optimization.

\subsection{Legacy model}

The flash smelting furnace process has been modelled in HSC-Sim as a static division process with empirical knowledge controlling parts of the division coefficients. The implementation is a spreadsheet-like division calculation with iterative calculation to fulfill constraints derived from empirical and physical knowledge.

The model consists of three main spreadsheets; Input, Distributions and Output, each containing 146-424 rows and 68 columns. The Input sheet is sparsely filled with element mass flows and describes how input compounds in different streams are broken up to elements according to chemical molar consistency. The Distributions sheet is sparsely filled with distribution coefficients dividing element mass flow into compounds for different output streams partly according to chemical reactions. The Output sheet is filled with corresponding element mass flows that build up the output compounds in different output streams. Additionally, to the three main spread sheets, a Controls sheet includes 27 empirical process observations that must be fulfilled in the stationary state.

In principle, the distribution from input compounds to output compound is built up around how the main elements copper $(\mathrm{Cu})$ and iron $(\mathrm{Fe})$ is distributed between compounds in the output streams. The chemical reactions requre assisting element as oxygen (O) and silicon $(\mathrm{Si})$ which are brought in as floating elements. Sulphur (S) is partly handled as a main element and partly as an assisting element. As a result the model consists of some fixed distribution coefficients, many coefficients which are iteratively adjusted to fulfill the empirical observations and numerous coefficients calculated as float according to corresponding chemical reactions or as rest for surplus elements. The model is thus a system of four spreadsheets with a large number of interconnected cells. An iterative routine is used to solve the distribution coefficients and thereby the element and compound streams in the stationary state.

The calculation is very useful for off-line strategic planning of metal processing. The calculation is, however, too slow for real-time process optimization. 


\subsection{Method for derivation of fast calculating model}

In general, the objective for the study was to find a method for converting iterative output controlled balance models to directly calculating models suitable for process scheduling. The basic idea was to form a symbolic equation group based on the flash smelting furnace HSC-Sim model and to solve this group analytically with symbolic computation to achieve causal outputs as direct functions of inputs. The solution is possible due to empirical knowledge included in the Controls sheet of the FSF HSC-Sim model.

Thus, the task was to write a fully parametrized equation group based on the FSF HSC-Sim model where the equations are based on the equations of empirical knowledge in the Controls sheet. The model is in this analytic approach simplified. The input elements include only the main elements; copper $(\mathrm{Cu})$, iron $(\mathrm{Fe})$, nitrogen $(\mathrm{N})$, oxygen $(\mathrm{O})$, sulphur $(\mathrm{S})$, silicon $(\mathrm{Si})$ and other content $(\mathrm{Ot})$. The distribution of the elements between the output streams, which are settler gas, settler fume, settler dust, slag and matte, is fully in line with the FSF HSC-Sim model. The eight equations determining empirical knowledge regarding the main elements was chosen as base for the equations. To enable an analytic solution with the symbolic software the equation group has to be exactly determined.

The equation group formulation starts with defining all basic variables as symbolic variables. This example included 7 element mass flows, 23 distribution coefficients for element distribution to output streams and 41 distribution coefficients for element distribution into compounds in the different output streams. The main formulation work is to define the relationship between these variables with emphasis on the formulation of the float and rest variables. Here, this part required about 75 definitions. After these definitions, the output compounds can be formulated. Afterwards, the final equations based on the empirical knowledge in the Controls sheets can be written. To ease the derivation of the analytic solution of the software the nonlinearities in the empirical knowledge were linearized. The same variables as the manipulated variables in the iterative solution of HSC-Sim model were chosen as variables for the calculation to solve. They were; distribution coefficient for $\mathrm{Fe}$ to matte, distribution coefficient for $\mathrm{Fe}$ in slag to $\mathrm{FeS}$, distribution coefficient for $\mathrm{Cu}$ to slag, distribution coefficient for $\mathrm{Fe}$ in matte to $\mathrm{Fe}_{3} \mathrm{O}_{4}$, Ot to matte, $\mathrm{Si}$ input stream, $\mathrm{O}$ input stream and distribution coefficient for $\mathrm{Fe}$ in slag to $\mathrm{Fe}_{3} \mathrm{O}_{4}$.

This study utilizes the Symbolic Math Toolbox in the Matlab software. With the relationships concerning use of oxygen still undefined, the solver managed to achieve a fully symbolical solution in around five minutes with a laptop. When oxygen is taken into account, the solver has been forced to settle for a numeric approximation, which still includes all the variables in an appropriate manner. The length of the analytic solutions is over 25 000 characters. The solutions are at this stage provided with the values of the fixed variables. The last task of the program is to produce usable functions of the long analytic solutions.

\section{Model Validation and Discussion}

Model validation is performed to ensure usability of the model in real-time process optimization and scheduling. As copper content in matte is a good measure of the process state, the validation is performed at varying matte copper percentage.

\subsection{Similarity to legacy model}

Figure 2 shows a comparison between the analytical direct solution results, with the blue line, and iteratively calculated HSC-Sim results, red line, as function of matte copper percentage.

The cause for the differences is the fact that the analytically solved model is a simplified model of the process including only the main elements. E.g. both silicon and oxygen is consumed by other minor compounds which are not included in the model. The difference is mainly a shift of magnitude which can easily be compensated by a term proportional to the total concentrate flow. With this compensation the analytically solved model is adequate for the on-line utilization.

\subsection{Calculation time}

As the optimization and scheduling algorithm calls the model hundreds of times per second the calculation time has to be short. A test function call from Matlab showed that the execution time is only some milliseconds for calls of two to eight variables, which is sufficient for the on-line utilization. The calculation time for the iterative solution of the HSC-Sim model is tens of seconds.

\section{Model Utilization}

The directly calculating model of the flash smelting furnace process will be utilized in scheduling of a copper production line to optimize production and costs. When solving the equation group the solvable variables can be freely chosen. There are two evident ways of model formulation that can be utilized.

\subsection{Direct input output}

A natural solution would be to form a direct input output model to mimic the real smelting process. Figure 3 represents a scheduling structure that utilizes the input output model. As scheduling is a high level task whose interests are in production rate and oxidation level in first stage smelting, a lower level control structure has to deal with the unit control of the flash smelting furnace. This is shown as feedback control of the open 

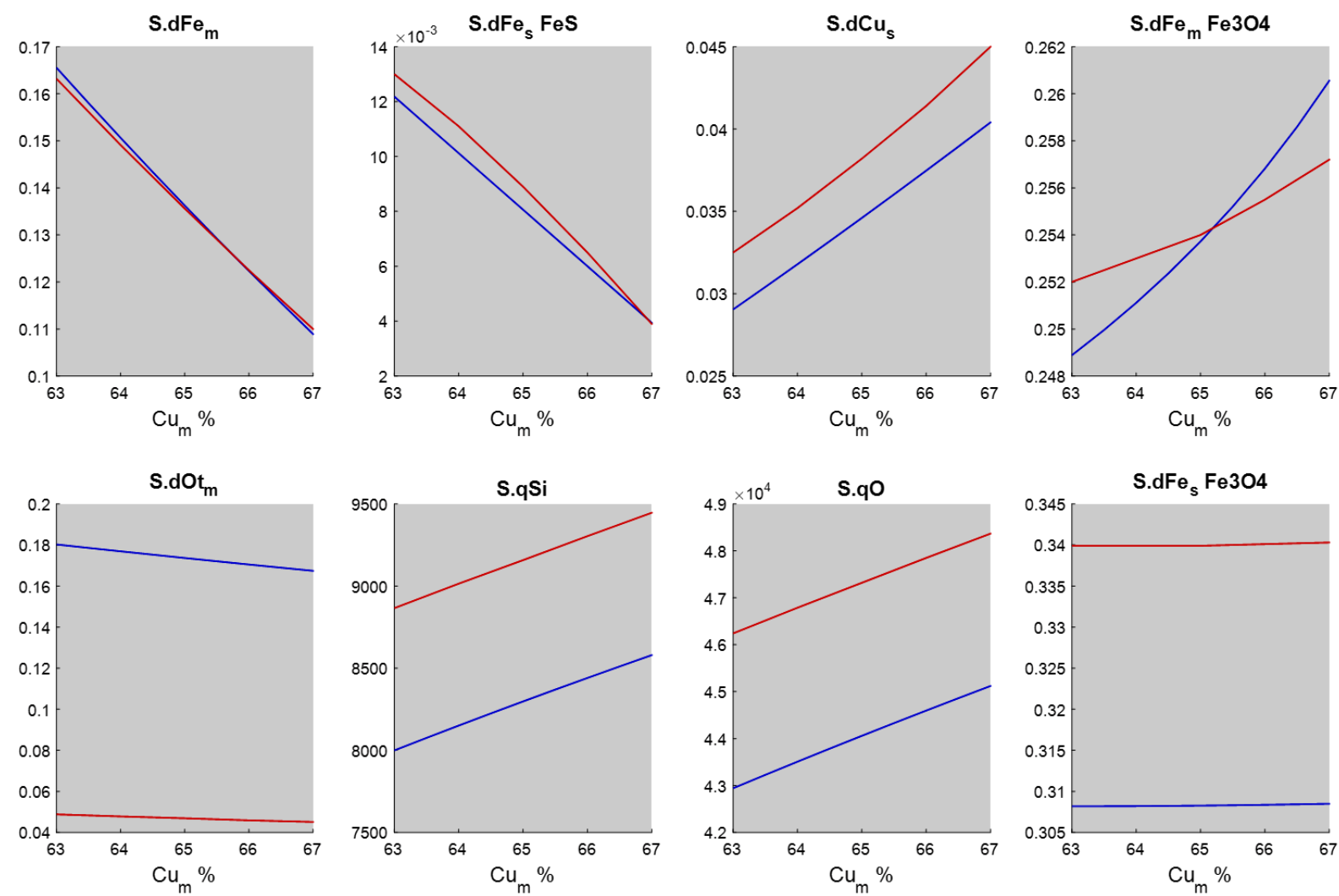

Figure 2. A comparison between analytical solution results with blue line and iteratively calculated HSC-Sim results with red line.

loop model. In practice, this could be a sub optimization task for the scheduling routine.

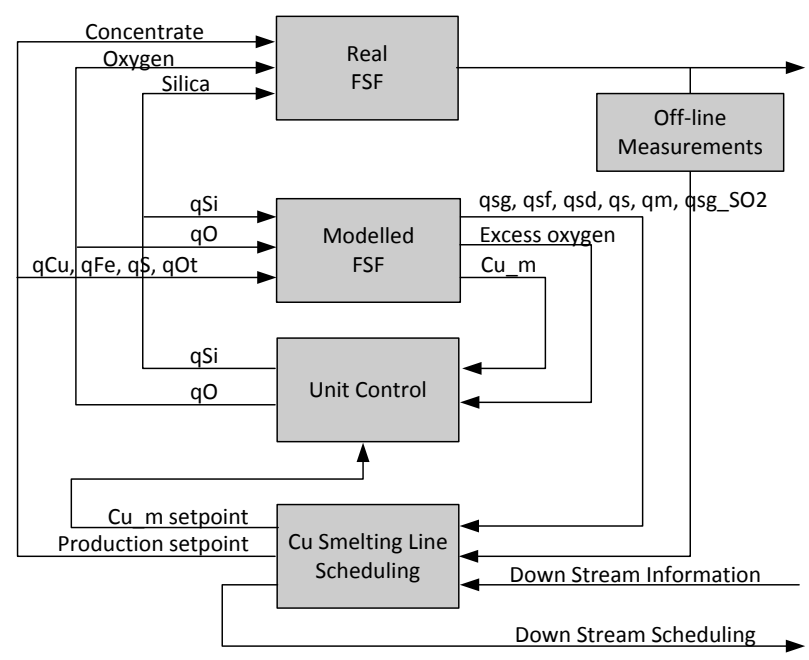

Figure 3. Direct input output model utilized in scheduling.

\subsection{Closed analytic solution}

To enhance the direct scheduling interests, the required control variables can directly be chosen as solvable variables in the equation group. The static model allows us to utilize a closed analytic solution whose scheduling structure is clear and shown in Figure 4. This direct solution will not need the sub optimization. Feedback from the off-line measurements compensates for model inaccuracy.

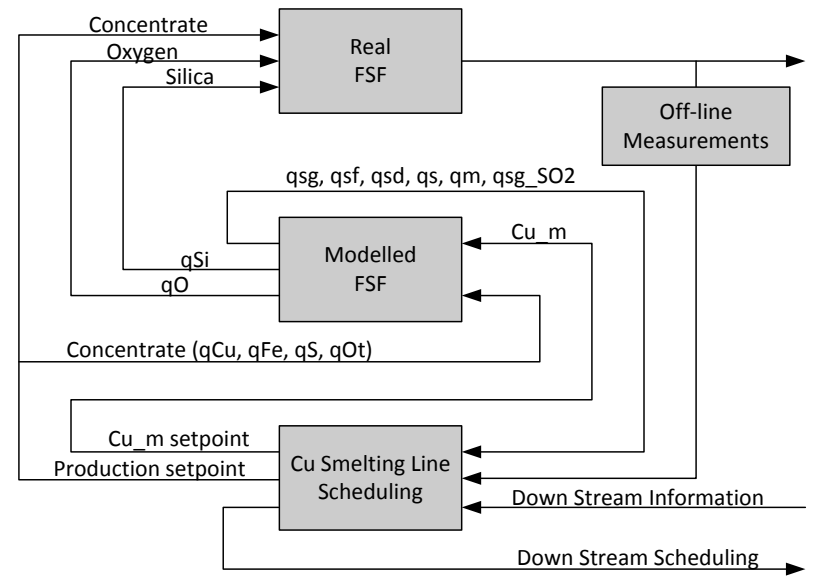

Figure 4. Closed analytic solution utilized in scheduling.

\section{Conclusions}

The objective of this study was to develop a method for converting iterative output controlled balance models to directly calculating models for process optimization and 
scheduling. This method was used in the case of a flash smelting furnace, previously modelled in HSC-Sim. The fast calculating model is to be used in optimization of the total production line operation.

The method consisted of formulating an equation group based on the constrained FSF HSC-Sim model and solving the unknown parameters and static states with use of a symbolic calculation software. The study was successful even if it requires careful formulation work and the solution matched the solution of the original model. The equation group should be fully determined to enable a solution. The solution was implemented as a direct calculation function whose calculation time fulfilled the requirements for scheduling use.

The advantage with the approach is that even though the length of the generated functions disables model maintenance in function form, functions can easily be recalculated after updates in the HSC-Sim model are done. The modelling method has shown to be a powerful general way of converting complex iteratively solvable models to fast directly calculating models for utilization in process optimization and different operator advisory systems.

The presented demonstration model did not include an energy balance and thereby the amount of nitrogen (N) feed is kept constant even if the nitrogen feed is in practice the means to affect process temperature. The legacy model is built on the assumption that temperature is on normal level which enables a mass balance without temperature dependency. The energy balance will be included in future work.

\section{Acknowledgements}

This work was carried out in the SIMP research program coordinated by the Finnish Metals and Engineering Competence Cluster (FIMECC) Ltd. The support is gratefully acknowledged. The authors are also grateful for the process expert knowledge and good working possibilities provided by Petri Latostenmaa and Ville Naakka at Boliden Harjavalta.

\section{References}

W. Belkhir, N. Ratier, D. D. Nguyen, B. Yang, M. Lenczner, F. Zamkotsian and H. Cirstea. Towards an automatic tool for multi-scale model derivation illustrated with a micromirror array. In 17th International Symposium on Symbolic and Numeric Algorithms for Scientific Computing 2015, pages 47-54. doi 10.1109/synasc.2015.17.

Boliden.

$$
\text { Available }
$$

via

www.boliden.com/Documents/Press/Publications/Place\%2 0broschures/boliden-harjavalta-en.pdf. [accessed June 23, 2016].

I. Harjunkoski and R. Bauer. Configurable Scheduling Solution using Flexible Heuristics. In Proceedings of the 26th European Symposium on Computer Aided Process Engineering - ESCAPE 26 2016, pages 2362-2366.
T. Korpela, T. Björkqvist, Y. Majanne and P. Lautala. Online Monitoring of Flue Gas Emissions in Power Plants having Multiple Fuels. IFAC-PapersOnLine, 19(1): 1355-1360, 2014. doi: 10.3182/20140824-6-ZA-1003.01913.

J. Lin and C. Chen. Computer-aided-symbolic dynamic modeling for Stewart-platform manipulator. Robotica, 27: 331-341, 2009.

Maplesoft. Available via www.maplesoft.com/solutions/education/. [accessed June 23, 2016].

Mathworks. Available via www.mathworks.com/. [accessed June 23, 2016].

S. A. A. Moosavian and E. Papadopoulos. Explicit dynamics of space free-flyers with multiple manipulators via SPACEMAPLE. Adv. Rob., 18: 223-244, 2004.

Outotec. Available via www.outotec.com/en/Products-services/HSC-Chemistry/Calculation-modules/Sim-process-simulation/. [accessed June 23, 2016].

D. Pelusi. PID and intelligent controllers for optimal timing performances of industrial actuators. International Journal of Simulation: Systems, Science and Technology, 13(2): 6571, 2012.

D. Pelusi. Improving settling and rise times of controllers via intelligent algorithms (2012). In Proceedings - 2012, 14th International Conference on Modelling and Simulation, UKSim 2012, art. no. 6205447, pages 187-192.

M. E. Schlesinger, M. J. King, K. C. Sole and W. G. Davenport (2011). Extractive metallurgy of copper. Elsevier.

C. Touretzky, I. Harjunkoski and M. Baldea. A Framework for Integrated Scheduling and Control using Discrete-Time Dynamic Process Models. In Proceedings of the $26^{\text {th }}$ European Symposium on Computer Aided Process Engineering - ESCAPE 26 2016, pages 601-606.

Wolfram. Available via www.wolfram.com/mathematica/. [accessed June 23, 2016]

V. Yakhno and M. Altunkaynak. A polynomial approach to determine the time-dependent electric and magnetic fields in anisotropic materials by symbolic computations. COMPEL - the International Journal for Computation and Mathematics in Electrical and Electronic Engineering, 35: 1179-1202, 2016. 